\title{
Aspectos clínicos e epidemiológicos da leishmaniose visceral em menores de 15 anos no estado do Rio Grande do Norte, Brasil
}

\author{
Clinical and epidemiological aspects of visceral leishmaniasis in \\ children up to 15 years of age in Rio Grande do Norte state, Brazil
}

\author{
Isabelle Ribeiro Barbosa ${ }^{1}$, Íris do Céu Clara Costa ${ }^{2}$ \\ ${ }^{1}$ Farmacêutica. Doutoranda do Programa de Pós-Graduação em Saúde Coletiva da Universidade Federal do Rio Grande do Norte. \\ 2 Odontóloga. Doutora em Odontologia Preventiva e Social. Professora Associada II do Departamento de Odontologia da \\ Universidade Federal do Rio Grande do Norte.
}

\begin{abstract}
RESUMO
Objetivos: Descrever as características clínicas e epidemiológicas de crianças e adolescentes com leishmaniose visceral (LV) no estado do Rio Grande do Norte, Brasil.

Métodos: Foi realizado um estudo descritivo de casos autóctones de LV em indivíduos de 0 a 15 anos de idade. Foram analisadas as informações referentes ao período de janeiro de 2007 a dezembro de 2011, constantes no Sistema de Informações de Agravos de Notificações do Ministério da Saúde.

Resultados: Foram identificadas 291 crianças e adolescentes com LV, sendo 50,8\% do sexo masculino. A faixa etária de maior incidência da doença foi a de menores de 5 anos $(72,5 \%$ dos casos). Verificou-se que $16,1 \%$ dessas crianças residiam em Natal, capital do estado, e 40,9\% procediam de quatro municípios do RN; 70,4\% eram originárias da zona urbana. A principal manifestação clínica foi a febre $(76,9 \%)$ e os principais achados ao exame físico foram palidez $(68 \%)$, esplenomegalia $(67,7 \%)$ e hepatomegalia $(63,9 \%)$. O tratamento mais utilizado foi antimoniato de $\mathrm{N}$-metil glucamina (61\%), seguido por anfotericina B lipossomal $(5,77 \%)$ e anfotericina B convencional $(1,44 \%)$. O tempo médio entre a ocorrência dos primeiros sintomas e o início do tratamento foi de 32,5 dias; $69,4 \%$ dos pacientes evoluíram para cura e 3,8\% evoluíram para óbito.

Conclusões: A incidência de LV no grupo de menores de 15 anos no Rio Grande do Norte, nos cinco anos estudados, foi superior à observada na população em geral no Brasil. O maior número de casos ocorreu em áreas urbanas. Em muitos casos houve um grande lapso de tempo entre o aparecimento dos sintomas e o início do tratamento. Sugere-se uma observação mais dirigida por parte dos profissionais de saúde, visando ao reconhecimento precoce da doença nas crianças, possibilitando tratamento adequado e evitando complicações.
\end{abstract}

DESCRITORES: LEISHMANIOSE VISCERAL/epidemiologia; ZOONOSES; DOENÇAS TRANSMISSÍVEIS; VIGILÂNCIA EPIDEMIOLÓGICA; CRIANÇA; ADOLESCENTE.

\begin{abstract}
Aims: To describe the clinical and epidemiological characteristics of children and adolescents with visceral leishmaniasis (VL) in the state of Rio Grande do Norte (RN), Brazil.

Methods: A descriptive study of individuals 0-15 years with a diagnosis of autochthonous cases of VL was performed. We analyzed information for the period from January 2007 to December 2011, contained in the Disease Notifications Information System of the Brazilian Ministry of Health.

Results: We identified 291 children and adolescents with VL, of whom $50.8 \%$ were male. The age group with the highest incidence of the disease was that of children under 5 years $(72.5 \%$ of cases). It was found that $16.1 \%$ of these children lived in Natal (the state capital) and 40.9\% were from four municipalities in RN; $70.4 \%$ were from the urban area. The main clinical manifestation was fever $(76.9 \%)$ and the major physical findings were pallor $(68 \%)$, splenomegaly $(67.7 \%)$ and hepatomegaly $(63.9 \%)$. The most commonly used treatment was N-methylglucamine antimoniate (61\%), followed by liposomal amphotericin B (5.77\%) and conventional amphotericin B (1.44\%). The average time between the occurrence of the first symptoms and the beginning of treatment was 32.5 days; $69.4 \%$ of subjects patients achieved cure and $3.8 \%$ died.

Conclusions: The incidence of LV in the group of children under 15 years of age in Rio Grande do Norte, in the five studied years, was higher than that observed in the general population in Brazil. The largest number of cases occurred in urban areas. In many cases there was a considerable time gap between onset of symptoms and start of treatment. It is suggested a more targeted observation on the part of health professionals, aiming at early recognition of the disease in children, allowing appropriate treatment and preventing complications.
\end{abstract}

KEY WORDS: LEISHMANIASIS, VISCERAL/epidemiology; ZOONOSES; COMMUNICABLE DISEASES; EPIDEMIOLOGICAL SURVEILLANCE; CHILD; ADOLESCENT. 


\section{INTRODUÇÃO}

A leishmaniose visceral (LV), também conhecida por calazar, é uma zoonose causada por protozoários do gênero Leishmania. No Brasil, é causada pela espécie Leishmania chagasi, transmitida aos seres humanos através da picada de fêmeas infectadas do mosquito flebotomíneo Lutzomyia longipalpis. Essa doença continua a ser um grande desafio à saúde pública, pela frequência com que ocorre e pela morbidade e mortalidade que ocasiona. ${ }^{1,2}$

A LV é endêmica em 70 países de áreas tropicais e subtropicais de quatro continentes. Estima-se que ocorram a cada ano 500.000 novos casos e 50.000 mortes em todo o mundo. Os principais focos de infecção humana estão localizados no sudoeste asiático (300.000 casos em 2006), no oeste da África (cerca de 30.000 casos em 2006) e no continente americano (4.000 casos em 2006). ${ }^{1,3} \mathrm{Na}$ América Latina, onde é denominada LV americana, foi descrita em 12 países, sendo que $90 \%$ dos casos ocorrem no Brasil. Na década de 1990 , aproximadamente $80 \%$ dos casos notificados de LV ocorriam na região Nordeste do país. ${ }^{4}$

Antes restrita às áreas rurais do nordeste brasileiro, a LV avançou para a periferia das grandes cidades, alcançando inclusive regiões brasileiras antes indenes. Casos autóctones da doença já foram registrados em 19 dos 27 estados brasileiros. ${ }^{5}$ Acredita-se que a urbanização da LV resulta das alterações realizadas pelo homem no ambiente e dos fluxos migratórios das populações rurais para as periferias urbanas. Nessas áreas, coexistem as condições inadequadas de habitação, infraestrutura e saneamento e a mobilização e interação simultânea de reservatórios silvestres e cães infectados com L. chagasi nas zonas sem transmissão, mantendo as condições propícias para a multiplicação do vetor. ${ }^{6}$ Nas áreas periurbanas, os cães têm sido tradicionalmente considerados os principais reservatórios de L. chagasi. $^{7}$

Na maioria das áreas endêmicas no Brasil, a LV é mais frequente entre as crianças. Até os anos 1970, crianças com menos de 10 anos de idade responderam por $80 \%$ dos casos, sendo a faixa etária de crianças menores de 5 anos de idade a mais acometida (60\% do total). Atualmente, a prevalência tem diminuído entre as crianças menores de 10 anos de idade, agora representando cerca de $50 \%$ dos casos. ${ }^{8,9}$ A razão da maior suscetibilidade da criança está ligada a maior vulnerabilidade da resposta imune, provocada pela imaturidade da imunidade humoral e celular, e pela imunodepressão induzida pela desnutrição, situação frequente nas populações pobres do Nordeste, que representa um fator de predisposição para a infecção. ${ }^{10}$
As apresentações clínicas da LV variam desde formas assintomáticas até um quadro caracterizado por febre, anemia, hepatoesplenomegalia, manifestações hemorrágicas, linfadenomegalia, perda de peso, taquicardia, tosse seca e diarreia (estes dois últimos sintomas menos frequentes). A desnutrição ocorre com a progressão da doença, podendo manifestar-se por edema periférico, queda de cabelos e alterações de pele e das unhas. ${ }^{11}$ A identificação precoce das características clínicas e laboratoriais no primeiro atendimento ao paciente é de fundamental importância para se reduzir a mortalidade por meio da instituição de medidas terapêuticas e profiláticas eficazes em tempo oportuno..$^{1,12}$ Os esquemas tradicionais para o tratamento da LV são os antimoniais pentavalentes e a anfotericina B. Recentemente surgiram outros esquemas de tratamento, como a miltefosina (primeira droga oral), as formas lipídicas ou lipossomais da Anfotericina B e a Paramomicina. ${ }^{13}$

Atualmente, reconhece-se a necessidade de estudos que abordem o padrão de ocorrência de doenças e agravos endêmicos, especialmente nos grupos populacionais mais afetados, levando-se em conta que apesar de características comuns, podem adquirir aspectos específicos e peculiares. Esses estudos podem subsidiar o entendimento da dinâmica do agravo, bem como importantes ações de vigilância em saúde. No Brasil, principalmente na região Nordeste, foram realizados poucos estudos que abordam este tema, permanecendo as características ainda pouco esclarecidas. Neste contexto, propõe-se como objetivo deste estudo descrever as características clínicas e epidemiológicas dos casos diagnosticados de LV em crianças e adolescentes no estado do Rio Grande do Norte.

\section{MÉTODOS}

Realizou-se um estudo descritivo de abordagem quantitativa, que analisou dados secundários de casos autóctones de LV do estado do Rio Grande do Norte, registrados no Sistema de Informação de Agravos de Notificação (SINAN), do Ministério da Saúde, bem como os óbitos registrados no Sistema de Informação sobre Mortalidade (SIM). Foram utilizados também dados disponibilizados pela Subcoordenadoria de Vigilância Epidemiológica da Secretaria de Estado da Saúde Pública do Rio Grande do Norte (SUVIGE/SESAP/RN).

O estado do Rio Grande do Norte está localizado na região Nordeste do Brasil, na latitude $5^{\circ} 47^{\prime} 42^{\prime \prime} \mathrm{S}$ e longitude $35^{\circ} 12$ ' 32 '. O estado tem a área de $52.796,79 \mathrm{~km}^{2}$, representando $\mathbf{3 , 4 1 \%}$ da região Nordeste e $\mathbf{0 , 6 2} \%$ de todo o território brasileiro. Segundo o 
Instituto Brasileiro de Geografia e Estatística (IBGE), sua população foi estimada em 3.168.027 habitantes para o ano de 2010, sendo a população de 0 a 15 anos composta por 850.425 habitantes. Possui 167 municípios divididos administrativamente em sete regionais de saúde.

Analisou-se a distribuição dos casos de acordo com as principais variáveis epidemiológicas e clínicas descritas nas fichas de notificação/investigação do SINAN, a saber: idade, sexo, raça, tipo de entrada (caso novo/recidiva), zona de residência (rural/urbana/ periurbana), procedência, sintomatologia, evolução, critérios de confirmação, tratamento, coinfecção com o HIV e taxa de letalidade. Os dados foram consolidados a partir da utilização do TabWin versão 3.6, que permitiu a análise estatística descritiva do estudo. As estimativas populacionais empregadas para o cálculo do coeficiente de incidência por 100.000 habitantes foram obtidas do Instituto Brasileiro de Geografia e Estatística (IBGE). Os dados foram obtidos de fonte secundária de consulta pública, sem a identificação nominal dos pacientes. Foram respeitados todos os aspectos éticos da Resolução № 196 de 10 de outubro de 1996 do Conselho Nacional de Saúde.

\section{RESULTADOS}

No período de janeiro de 2007 a dezembro de 2011 foram diagnosticados 291 casos de LV em menores de 15 anos no estado do Rio Grande do Norte. O ano de 2011 foi o de maior incidência ( 8,73 casos/100mil habitantes), seguido do ano de 2008 (6,62 casos/100mil habitantes). $\mathrm{O}$ ano de 2009 foi o de menor incidência (5,65 casos/ 100 mil habitantes). Foram registrados 11 óbitos por LV, sendo um óbito em 2007, dois em 2008, três em 2009, três em 2010 e dois em 2011. A taxa de letalidade variou entre $1,85 \%$ (em 2007) a 5,88\% (em 2009) (Figura 1).
A distribuição por sexo mostrou 148 casos $(50,8 \%)$ em meninos. A faixa etária com a maior ocorrência de casos foi a de menores de cinco anos $(72,3 \%)$, merecendo destaque também a faixa etária de menores de um ano, que teve o percentual de $16 \%$ dos casos. A maior parte dos casos acometeu indivíduos de cor preta e parda $(62,2 \%)$. Os pacientes eram oriundos em sua maioria $(72,4 \%)$ de zonas urbanas e periurbanas (Tabela 1).

Tabela 1. Variáveis demográficas de 291 casos de leishmaniose visceral em menores de 15 anos. Rio Grande do Norte, Brasil, 2007-2011.

\begin{tabular}{lcc}
\hline \multicolumn{1}{c}{ Variável } & n & \% \\
\hline Faixa etária & 47 & 16,0 \\
Menor de 01 ano & 164 & 56,3 \\
01 a 05 anos & 52 & 17,8 \\
06 a 10 anos & 28 & 9,6 \\
11 a 15 anos & & \\
Raça/cor & 32 & 10,9 \\
Ignorada & 170 & 58,4 \\
Parda & 75 & 25,7 \\
Branca & 11 & 3,8 \\
Preta & 2 & 0,68 \\
Amarela & 1 & 0,3 \\
Indígena & & \\
Sexo & 148 & 50,8 \\
Masculino & 143 & 49,2 \\
Feminino & & 2,0 \\
Zona de residência & 6 & 2,0 \\
Ignorada & 205 & 20,4 \\
Urbana & 74 & \\
Rural & 6 & 2,4 \\
Periurbana & & \\
\hline
\end{tabular}

Fontes: Sistema de Informação de Agravos de Notificação (SINAN), Ministério da Saúde; Secretaria de Estado da Saúde Pública do Rio Grande do Norte.

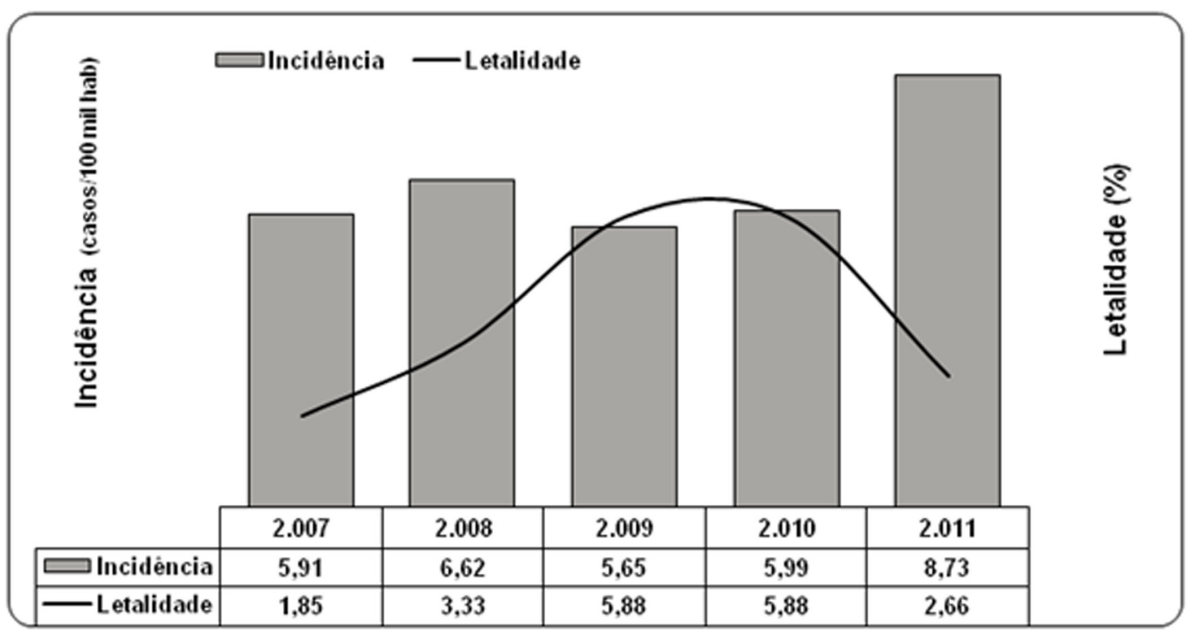

Figura 1. Incidência anual e taxa de letalidade por leishmaniose visceral em crianças e adolescentes de até 15 anos no estado do Rio Grande do Norte, Brasil, 2007-2011.

Fontes: Sistema de Informação de Agravos de Notificação (SINAN), Ministério da Saúde; Secretaria de Estado da Saúde Pública do Rio Grande do Norte; e Sistema de Informações sobre Mortalidade (SIM), DATASUS. 
Com referência às principais manifestações clínicas apresentadas, foram mais frequentes: febre $(76,9 \%)$, palidez $(68,0 \%)$, esplenomegalia $(67,7 \%)$ e hepatomegalia $(63,9 \%)$. O quadro infeccioso ocorreu em 15,1\% dos indivíduos (Tabela 2). O tempo decorrido entre o aparecimento dos primeiros sintomas e o início do tratamento variou de 1 a 189 dias, com média de 32,5 dias (desvio padrão $\pm 30,55$ dias), sendo que $19 \%$ dos pacientes iniciaram o tratamento após 50 dias do início dos sintomas.

Tabela 2. Frequência de manifestações clínicas em 291 casos de leishmaniose visceral em menores de 15 anos. Rio Grande do Norte, Brasil, 2007-2011.

\begin{tabular}{lcc}
\hline \multicolumn{1}{c}{ Manifestação clínica } & $\mathbf{N}^{*}$ & $\mathbf{\% *}^{*}$ \\
\hline Febre & 224 & 76,9 \\
Palidez & 198 & 68,0 \\
Esplenomegalia & 197 & 67,7 \\
Hepatomegalia & 186 & 63,9 \\
Emagrecimento & 132 & 45,3 \\
Fraqueza & 128 & 43,9 \\
Tosse e/ou diarreia & 102 & 35,0 \\
Edema & 66 & 22,6 \\
Icterícia & 47 & 16,1 \\
Quadro infeccioso & 44 & 15,1 \\
Hemorragia & 22 & 7,5 \\
\hline
\end{tabular}

* A maioria dos pacientes apresentava mais de uma manifestação.

Fontes: Sistema de Informação de Agravos de Notificação (SINAN), Ministério da Saúde; Secretaria de Estado da Saúde Pública do Rio Grande do Norte.

Verificou-se que $74,6 \%$ eram casos novos e $2 \%$ de recidiva; $69,4 \%$ evoluíram para cura, e 11 pacientes evoluíram para óbito, perfazendo a taxa de letalidade geral para o período de $3,8 \%$. Oito pacientes $(2,7 \%)$ estavam coinfectados com HIV. Quanto aos critérios de confirmação dos casos, o critério laboratorial foi o mais utilizado $(69,1 \%)$, incluindo o diagnóstico parasitológico em $48,79 \%$, seguido do critério imunológico pela utilização do antígeno recombinante rK39, no qual $36,1 \%$ das amostras foram reagentes, e pela imunofluorescência indireta, reagente em 12,3\% dos casos. O tratamento de escolha foi o antimoniato de N-metil glucamina (61\%). A anfotericina B convencional foi utilizada em $1,44 \%$ dos casos e a anfotericina B lipossomal em 5,77\%. A Tabela 3 mostra as características clínicas e laboratoriais dos 291 pacientes.

Considerando os municípios com o maior número de notificações de casos de LV em menores de 15 anos, o município de Mossoró obteve o maior número de registros (64 casos); o município de Açu registrou 27 casos. Ambos estão localizados no Oeste Potiguar. O município de Natal, a capital do estado, registrou 47 casos, seguido de Extremoz (14 casos), Nísia Floresta (14 casos) e Parnamirim (oito casos), todos localizados na região metropolitana. Os municípios de Equador e Major Sales não registraram casos no período; 31 municípios registraram um caso cada, nos cinco anos estudados.

Tabela 3. Características clínicas e laboratoriais de 291 casos de leishmaniose visceral em menores de 15 anos no estado do Rio Grande do Norte, Brasil, 2007-2011.

\begin{tabular}{|c|c|c|}
\hline Característica & $\mathbf{n}$ & $\%$ \\
\hline \multicolumn{3}{|l|}{ Tipo de entrada no sistema } \\
\hline Ignorado & 68 & 23,4 \\
\hline Caso novo & 217 & 74,6 \\
\hline Recidiva & 6 & 2,0 \\
\hline \multicolumn{3}{|l|}{ Critério de confirmação } \\
\hline Ignorado & 71 & 24,4 \\
\hline Laboratorial & 201 & 69,1 \\
\hline Clínico-epidemiológico & 19 & 6,5 \\
\hline \multicolumn{3}{|l|}{ Evolução } \\
\hline Ignorado & 70 & 24,1 \\
\hline Cura & 202 & 69,4 \\
\hline Óbito por leishmaniose visceral & 11 & 3,8 \\
\hline Transferência & 8 & 2,7 \\
\hline \multicolumn{3}{|l|}{ Coinfecção com o HIV } \\
\hline Ignorado & 115 & 39,6 \\
\hline Sim & 8 & 2,7 \\
\hline Não & 168 & 57,7 \\
\hline \multicolumn{3}{|l|}{ Droga inicial } \\
\hline Ignorado & 82 & 28,1 \\
\hline $\begin{array}{l}\text { Antimonial pentavalente } \\
\text { (antimoniato de N-metil glucamina) }\end{array}$ & 167 & 57,4 \\
\hline Anfotericina $b$ & 4 & 1,4 \\
\hline Anfotericina $\mathrm{b}$ lipossomal & 16 & 5,4 \\
\hline Outras drogas & 5 & 1,7 \\
\hline \multicolumn{3}{|l|}{ Diagnóstico parasitológico } \\
\hline Positivo & 142 & 48,79 \\
\hline Negativo ou não realizado & 149 & 51,21 \\
\hline \multicolumn{3}{|l|}{ Diagnóstico Imunológico (RIFI) } \\
\hline Positivo & 36 & 12,3 \\
\hline Negativo ou não realizado & 255 & 87,7 \\
\hline \multicolumn{3}{|l|}{ Diagnóstico Imunológico utilizando o rK39 } \\
\hline Positivo & 105 & 36,1 \\
\hline Negativo ou não realizado & 186 & 63,9 \\
\hline
\end{tabular}

* RIFI: reação de imunofluorescência indireta.

+ rK39: antígeno recombinante de 298 aminoácidos com peso molecular de 39 kilodaltons (kDA).

Fontes: Sistema de Informação de Agravos de Notificação (SINAN), Ministério da Saúde; Secretaria de Estado da Saúde Pública do Rio Grande do Norte.

\section{DISCUSSÃO}

Uma importante característica da LV no Brasil é que a alta incidência da doença na população infantil vem se mantendo ao longo dos anos..$^{14}$ Neste estudo, a incidência da doença no grupo de menores de 15 
anos no estado do Rio Grande do Norte foi superior à incidência observada para a população em geral para todo o Brasil, que nos últimos 10 anos registrou a média anual de 3.379 casos de LV e a incidência de 1,9 casos por 100.000 habitantes. ${ }^{9}$

A frequência da doença em menores de cinco anos verificada no presente estudo também foi observada no estudo de Xavier-Gomes et al., ${ }^{15}$ no qual $74,5 \%$ das crianças com LV tinham menos que cinco anos de idade, e no estudo de Pedrosa e Rocha, ${ }^{16}$ que observaram que $55,3 \%$ estavam abaixo dos cinco anos de idade. No estudo de Queiroz et al., ${ }^{14}$ a LV também predominou nos primeiros cinco anos de vida, faixa etária de $68,2 \%$ dos doentes. O percentual de casos observados neste estudo em menores de um ano foi inferior ao registrado no estudo de Barreto, ${ }^{17}$ no qual $44 \%$ das crianças infectadas estavam nessa faixa etária; o estudo de Dionísio et al., ${ }^{18}$ relatando uma experiência em um centro pediátrico de referência em Portugal, observou que $71 \%$ das crianças infectadas tinham idade inferior a dois anos.

A idade é um importante fator de risco para a ocorrência da LV humana, que atinge principalmente as crianças, cuja susceptibilidade é aumentada pela imaturidade da imunidade celular e pelo estado imunodepressivo agravado geralmente pela desnutrição. A elevada incidência da doença nesta faixa etária sugere a ocorrência da infecção nos ambientes peridomiciliar e intradomiciliar. ${ }^{19,20}$

Conforme o Ministério da Saúde, ${ }^{21}$ não existe diferença de susceptibilidade para LV entre os sexos. $O$ presente estudo identificou uma proporção entre sexo masculino/feminino de 1,03/1. Pastorino et al. ${ }^{11}$ descrevem uma proporção de 1,29 meninos/1 menina em 78 crianças com LV procedentes de vários estados brasileiros; Pedrosa et al. ${ }^{16}$ encontraram $58 \%$ do sexo masculino entre 530 casos de LV em menores de 15 anos; Oliveira et al., ${ }^{22}$ estudando a LV em Três Lagoas, Mato Grosso do Sul, encontraram 71,1\% de pacientes do sexo masculino, incluindo todas as faixas etárias (os homens preponderaram na faixa etária economicamente ativa); e Alvarenga et al. ${ }^{23}$ relatam $74,7 \%$ de homens entre 76 pacientes adultos em Mato Grosso do Sul. Entretanto, Santana et al., ${ }^{10}$ em São Luís do Maranhão, encontraram 22 meninas (56\%) entre 39 crianças com LV; Queiroz et al., ${ }^{14}$ entre 431 crianças diagnosticadas com LV no estado de Pernambuco, encontraram $50,3 \%$ de meninas; e, segundo o estudo de XavierGomes et al., ${ }^{15}$ entre 51 crianças procedentes de vários municípios do norte de Minas Gerais, hospitalizadas por $\mathrm{LV}, 51 \%$ eram do sexo feminino.

A LV pode modificar a progressão da doença pelo HIV, já que a Leishmania é um parasito intrace- lular obrigatório de células do sistema fagocitário mononuclear, e sua presença determina uma supressão reversível e específica da imunidade mediada por células. Por outro lado, a imunodepressão causada pelo HIV facilita a progressão da LV. ${ }^{9}$ Neste estudo, o percentual de crianças e adolescentes coinfectadas com o HIV foi inferior ao observado no estudo de Sousa-Gomes et al., ${ }^{24}$ que registrou um percentual de coinfecção de 8,3\% para indivíduos menores de 10 anos de idade notificados com LV no Brasil; da mesma forma, foi observado no estudo de Goes et al. ${ }^{3}$ o percentual de coinfecção de $6,9 \%$ em indivíduos de todas as faixas etárias em Aracaju, estado de Sergipe.

As manifestações clínicas mais frequentemente relatadas na admissão dos pacientes (febre, palidez, esplenomegalia, hepatomegalia, emagrecimento, fraqueza e tosse) também foram descritas por outros autores, com percentuais variáveis entre o achado de cada autor. ${ }^{3,11,14-16,25}$ Em zona endêmica, todo quadro clínico que se apresente com hepatoesplenomegalia febril deve ser investigado como quadro suspeito de LV. ${ }^{21}$ Achados com linfadenomegalia não foram descritos nos casos deste estudo, assim como em outros estudos realizados no Brasil., 22

Em relação ao diagnóstico parasitológico, no Brasil a positividade da microscopia direta em pacientes pediátricos tem sido de $69,3 \%$ a $81,2 \% .{ }^{15} \mathrm{~A}$ demonstração do parasito pode ser feita em material de biópsia ou punção aspirativa do baço, fígado, medula óssea ou linfonodos. A especificidade desses métodos é de $100 \%$, mas a sensibilidade é muito variável, pois a distribuição dos parasitos não é homogênea no mesmo tecido. ${ }^{5}$ Neste estudo a positividade do exame parasitológico foi de $48,79 \%$, semelhante aos $55 \%$ do estudo de Xavier-Gomes et al. ${ }^{14}$ e inferior à observada no estudo de Queiroz et al., ${ }^{15}$ no qual $75,5 \%$ das amostras foram positivas na pesquisa do parasita pelo mielograma, e no de Rey et al., ${ }^{25}$ no qual $73 \%$ das amostras apresentaram resultados positivos para formas amastigotas.

Antígenos recombinantes de Leishmania também têm sido utilizados como ferramenta para diagnóstico sorológico da infecção. O rK39 é uma sequência de 298 aminoácidos com peso molecular de 39 Kilodaltons (kDA) presente, predominantemente, em amastigotas de $L$. chagasi e $L$. donovani e tem sido usado como antígeno nesses testes. ${ }^{26}$ Como pode ser observado nos resultados dessa pesquisa, esse método laboratorial está sendo amplamente utilizado na prática clínica no estado do Rio Grande do Norte.

O exame imunológico mais utilizado no Brasil para essa finalidade é a reação de imunofluorescência 
indireta (RIFI). Na presença de dados clínicos e laboratoriais, um teste sorológico reagente reforça o diagnóstico de LV. Entretanto, um teste reagente na ausência de manifestações clínicas sugestivas de LV não autoriza o início do tratamento. ${ }^{21}$ Neste estudo, a positividade para o teste imunológico foi de $12,3 \%$, resultado inferior ao do estudo de Rigo et al., ${ }^{4}$ no qual a RIFI foi positiva em $87 \%$ dos casos.

Em relação ao início tratamento, verificou-se que praticamente um quinto dos pacientes iniciaram o tratamento após 50 dias do início dos sintomas. Alguns autores identificaram, como um dos sete fatores associados ao mau prognóstico da doença, tempo entre o início dos sintomas e primeira consulta médica acima de 56 dias. ${ }^{23,27}$ Segundo a Organização Mundial de Saúde, o início tardio da terapêutica específica é um fator que piora o prognóstico, e quanto mais precocemente for estabelecido o diagnóstico e instituído o tratamento, maior a chance de recuperação dos pacientes. ${ }^{1}$

No Brasil, apesar de não existir documentação da presença de cepas de L. chagasi resistentes in vitro aos antimoniais, recomenda-se o tratamento da LV com os derivados pentavalentes na dose de $20 \mathrm{mg}$ de antimônio pentavalente $\left(\mathrm{Sb}^{+5}\right)$ por $\mathrm{kg} / \mathrm{dia}$, com aplicação intravenosa ou intramuscular, por no mínimo 20 e no máximo 40 dias, utilizando-se o limite máximo de 2 a 3 ampolas por dia do produto, com bons índices de cura. Cada 1,5 g de antimoniato de N-metil glucamina corresponde a $405 \mathrm{mg}$ de $\mathrm{Sb}^{+5}$, dose contida em cada ampola de $5 \mathrm{ml}$ do medicamento distribuído pelo Ministério da Saúde. ${ }^{21}$

Nos últimos anos, a letalidade da LV vem aumentando gradativamente, passando de 3,6\% em 1994 para $6,7 \%$ em 2003, com um incremento de $85 \%$, e para $8,4 \%$ em $2004 .{ }^{21}$ A letalidade observada neste estudo foi inferior à do estudo de Queiroz et al. ${ }^{14}$ no qual a letalidade foi de $10,2 \%$, sendo que as principais causas imediatas de óbito foram atribuídas a infecções associadas, hemorragias e insuficiência hepática. A taxa de letalidade da LV observada em Campo Grande, Mato Grosso do Sul, foi de 18,4\%. ${ }^{23}$ No estudo de Rey et al., ${ }^{25}$ com crianças com LV americana admitidas no Hospital Infantil Albert Sabin, em Fortaleza, Ceará, a letalidade geral foi de 9,2\%, e em lactentes com menos de um ano foi de $21,2 \%$, sendo desnutrição, edema, sangramento, icterícia e infecção intercorrente os fatores relacionados com maior letalidade.

No estado do Rio Grande do Norte observa-se que o maior número de casos ocorreu em áreas mais urbanizadas. Nas últimas décadas ocorreram profundas mudanças na estrutura agrária do Brasil, que resultaram na migração de grande contingente populacional para os centros urbanos, ${ }^{28}$ resultando nas condições precárias de vida existentes nas periferias das grandes cidades. ${ }^{29}$ Os resultados obtidos sugerem que o estado do Rio Grande do Norte seja uma importante área endêmica para a LV no grupo de crianças e adolescentes. Embora essa elevada endemicidade não esteja distribuída uniformemente entre os municípios, destacam-se alguns municípios responsáveis pela maior carga da doença no estado, tornando-os importantes áreas para o monitoramento e controle dessa endemia.

Chama atenção o longo lapso de tempo entre o início dos sintomas e o tratamento, o que pode estar associado à taxa de letalidade descrita. Os dados sugerem a necessidade de uma observação mais minuciosa por parte dos profissionais de saúde, visando ao reconhecimento precoce e ao tratamento adequado da doença e de suas complicações, no intuito de diminuir as estatísticas de morbimortalidade infantil por LV.

Em áreas de baixa incidência de LV também é importante manter um elevado índice de suspeição clínica que permita um diagnóstico correto e tratamento em tempo oportuno. A LV deve ser sempre incluída no diagnóstico diferencial de pacientes com achados epidemiológicos, clínicos e laboratoriais característicos, mesmo nos pacientes oligossintomáticos. Diante desse quadro epidemiológico, é importante que as autoridades de saúde mantenham o monitoramento e a vigilância epidemiológica da LV no sentido de detectar e atuar frente a possíveis ocorrências de surtos de LV no estado do Rio Grande do Norte.

As estratégias recomendadas pelo Programa Nacional de Controle da LV são baseadas em um conjunto de medidas, tais como o diagnóstico precoce e tratamento dos casos humanos, inquérito sorológico canino e eutanásia de todos os cães soropositivos, pulverização sistemática de inseticida em domicílios e peridomicílio, e ações de educação em saúde e ambiental à população. ${ }^{30}$ É necessário fortalecer os programas de controle nessas áreas para que as medidas possam ser tomadas em conjunto e sejam eficazes na redução da incidência da doença.

Portanto, fazem-se necessários esforços conjuntos das diversas interfaces que compreendem áreas do conhecimento científico, somados aos serviços de saúde pública nos níveis estadual e municipal, buscando aperfeiçoar as ações de vigilância e controle da LV. Nesse contexto, instituições públicas e civis, assim como a própria sociedade, devem estar ativas e permanentemente inseridas no processo dinâmico, complexo e proativo das ações de controle. 


\section{REFERÊNCIAS}

1. Organización Panamericana de la Salud. Informe Final de la Reunión de Expertos OPS/OMS sobre Leishmaniasis Visceral en las Américas. OMS/OPS; 2006.

2. Amora SSA, Bevilaqua CML, Feijó FMC, et al. Sandflies (Psychodidae: Phlebotominae) survey in an urban transmission area of visceral leishmaniasis, Northeastern Brazil. Rev Bras Parasitol Vet. 2010;19:233-7.

3. Goes MAO, Melo CM, Jeraldo VLS. Time series of visceral leishmaniasis in Aracaju, state of Sergipe, Brazil (1999 to 2008): human and canine aspects. Rev Bras Epidemiol. 2012;15:298-307.

4. Rigo RS, Rigo L, Honer MR. Aspectos Clínicos e Laboratoriais na Leishmaniose Visceral Americana. J Bras Nefrol. 2009;31:48-54.

5. Gontijo CMF, Melo MN. Leishmaniose visceral no Brasil: quadro atual, desafios e perspectivas. Rev Bras Epidemiol. 2004;7:338-49.

6. Maia-Elkhoury ANS, Alves WA, Sousa-Gomes ML, et al. Visceral leishmaniasis in Brazil: trends and challenges. Cad Saúde Pública. 2008;24(12):2941-7.

7. Nascimento ELT, Martins GRM, Barbosa JD, et al. Forum: geographic spread and urbanization of visceral leishmaniasis in Brazil. Postscript: new challenges in the epidemiology of Leishmania chagasi infection. Cad Saúde Pública. 2008;24(12):2964-7.

8. Marzochi MCA, Fagundes A, Andrade MV, et al. Visceral leishmaniasis in Rio de Janeiro, Brazil: ecoepidemiological aspects and control. Rev Soc Bras Med Trop. 2009;42(5):570-80.

9. Brasil. Ministério da Saúde. Secretaria de Vigilância em Saúde. Guia de vigilância epidemiológica. Brasília (DF): Ministério da Saúde; 2009.

10. Santana JS, Silva AR, Cavalcante MNS, et al. Condições socioeconômicas, estado nutricional e consumo alimentar de crianças com Leishmaniose visceral atendidas em serviço público de saúde da cidade de São Luís, Maranhão, Brasil. Cad Pesq. 2009;16:55-62.

11. Pastorino AC, Jacob CMA, Oselka GW, et al. Leishmaniose visceral: aspectos clínicos e laboratoriais. J Pediatr. 2002; 78(2):120-7.

12. Medeiros FS, Tavares-Neto J, D'Oliveira Jr, et al. Alteraciones hepáticas em la Leishmaniasis Visceral (Kalazar) em niños: Revisión sistemática de la literatura. Acta Gastroenterol Latinoam. 2007;37:150-7.

13. Santos MA, Marques RC, Farias CA, et al. Predictors of an unsatisfactory response to pentavalent antimony in the treatment of American visceral leishmaniasis. Rev Soc Bras Med Trop. 2002;35(6):629-33.

14. Queiroz MJA, Alves JG, Correia JB. Leishmaniose visceral: características clínico-epidemiológicas em crianças de área endêmica. J Pediatr. 2004;80(2):141-6.
15. Xavier-Gomes LM, Costa WB, Prado PF, et al. Características clínicas e epidemiológicas da leishmaniose visceral em crianças internadas em um hospital universitário de referência no norte de Minas Gerais, Brasil. Rev Bras Epidemiol. 2009;12(4):549-55.

16. Pedrosa CMS, Rocha EMM. Aspectos clínicos e epidemiológicos da leishmaniose visceral em menores de 15 anos procedentes de Alagoas, Brasil. Rev Soc Bras Med Trop. 2004;37(4):300-4.

17. Barreto THP. Leishmaniose Visceral Grave em Crianças [Monografia de Especialização]. Campo Grande (MS): Universidade Federal do Mato Grosso do Sul; 2012.

18. Dionísio MT, Dias A, Rodrigues F, et al. Leishmaniose Visceral: Experiência de um centro pediátrico de referência 1990-2009. Acta Med Port. 2011;24:399-404.

19. Silva ES, Gontijo CMF, Pacheco RS, et al. Visceral leishmaniasis in the metropolitan region of Belo Horizonte, state of Minas Gerais, Brazil. Mem Inst Osw Cruz. 2001; 96:285-91.

20. Missawa NA, Borba JF. Leishmaniose visceral no município de Várzea Grande, Estado de Mato Grosso, no período de 1998 a 2007. Rev Soc Bras Med Trop. 2009;42(5):496-502.

21. Brasil. Ministério da Saúde. Manual de vigilância e controle da leishmaniose visceral. Brasília (DF): Ministério da Saúde; 2006.

22. Oliveira ALL, Paniago AMM, Dorval MEC, et al. Foco emergente de leishmaniose visceral em Mato Grosso do Sul. Rev Soc Bras Med Trop. 2006;39(5):446-50.

23. Alvarenga DG, Escalda PMF, Costa ASV, et al. Leishmaniose visceral: estudo retrospectivo de fatores associados à letalidade. Rev Soc Bras Med Trop. 2010;43(2):194-7.

24. Sousa-Gomes ML, Maia-Elkhoury NA, Pelissari DM, et al. Coinfecção Leishmania-HIV no Brasil: aspectos epidemiológicos, clínicos e laboratoriais. Epidemiol Serv Saúde. 2011;20(4):519-26.

25. Rey LC, Martins CV, Ribeiro HB, et al. Leishmaniose visceral americana (calazar) em crianças hospitalizadas de área endêmica. J Pediatr. 2005;81(1):73-8.

26. Cabral AP. Influência de fatores ambientais na leishmaniose visceral no Rio Grande do Norte. [dissertação]. Natal (RN): Universidade Federal do Rio Grande do Norte; 2007.

27. Abdelmoula MS. Visceral leishmaniasis in chidreen: prognostic factors. Tunis Med. 2003;81(5):535-9.

28. Scandar SAS, Silva RA, Cardoso-Junior RP, et al. Ocorrência de leishmaniose visceral americana na região de São José do Rio Preto, estado de São Paulo, Brasil. Bol Epidemiol Paul. 2011;8(88):13-22.

29. Bevilacqua PD, Paixão HH, Modena CM, Castro MCPS. Urbanização da leishmaniose visceral em Belo Horizonte. Arq Bras Med Vet Zootec. 2001;53(1):1-8.

30. Prado PF, Rocha MF, Souza JF, et al. Epidemiological aspects of human and canine visceral leishmaniasis in Montes Claros, State of Minas Gerais, Brazil, between 2007 and 2009. Rev Soc Bras Med Trop. 2011;44(5):561-6. 